\title{
Gesundheitsbezogene Register - gemeinsame Empfehlungen
}

Petra Buscha ${ }^{a}$ Regula Hellera, Angelina Hofstetterb, Stefanie Hostettler ${ }^{c}$, Esther Kraft ${ }^{c}$, Agnes Nienhaus ${ }^{d}$, Michelle Salathée

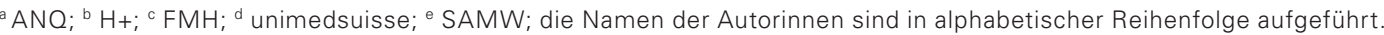

Register mit verlässlichen Daten werden im Gesundheitswesen immer wichtiger und mit den wachsenden Datenmengen zahlreicher. Um zur Qualitätssicherung beizutragen, haben die Organisationen FMH, ANQ, H+, SAMW und unimedsuisse gemeinsam Empfehlungen für den Aufbau und Betrieb von gesundheitsbezogenen Registern herausgegeben. Diese Empfehlungen enthalten Mindeststandards, unter anderem zu Datenschutz und Datenqualität.

Patientinnen und Patienten, Gesundheitspolitiker und Leistungserbringer haben ein berechtigtes Interesse an einer qualitativ hochstehenden und gleichzeitig finanzierbaren Gesundheitsversorgung. Gesundheitsbezogene Register übernehmen hierbei eine wichtige Funktion. Sie sind ein wichtiger Bestandteil der Qualitätssicherung, tragen bei zur Transparenz und Vergleichbarkeit medizinischer Leistungen, sind Grundlage für die klinische und epidemiologische Forschung und liefern wichtige Daten für die Gesundheitspolitik und -planung. Es gibt eine grosse Anzahl von Registern (http://www.fmh.ch/saqm/_ser vice/forum_medizinische_register.cfm), deren Aufgaben und Ziele je nach Anwendungsgebiet (und Rechtsgrundlage) unterschiedlich sind. Für die vorliegenden Empfehlungen wird ein gesundheitsbezogenes Register als systematische Sammlung von - gestützt auf definierte Kriterien erhobenen gesundheitsbezogenen Personendaten bezeichnet.

\section{Adressaten und Zielsetzung}

Die vorliegenden Empfehlungen richten sich in erster Linie an die für die Register verantwortlichen Leitungspersonen, deren Mitarbeitende und an die am Register Teilnehmenden. In zweiter Linie richten sie sich an die Leitungsgremien von Spitälern und weiteren Leistungserbringern, an Kranken- und Unfallversicherer und an politische Institutionen. Die Empfehlungen bieten Grundlagen, um bei laufenden sowie zukünftigen Registern deren Qualität zu überprüfen, und sollen sicherstellen, dass:
- gesundheitsbezogene Register über eine adäquate Struktur verfügen, damit sie ihren Auftrag erfüllen können;

- der Schutz der Persönlichkeitsrechte der Personen, deren gesundheitsbezogene Personendaten erfasst werden, sichergestellt ist;

- die Träger von Registerorganisationen sich daran orientieren können;

- die für das Register Verantwortlichen und die Mitarbeitenden über die notwendigen fachlichen inhaltlichen sowie technischen Kompetenzen verfügen;

- die dazu notwendigen personellen und finanziellen Ressourcen bereitgestellt sind;

- die Erhebung, Erfassung und Verwendung der Daten nach klar definierten Kriterien erfolgt;

- die Qualität der Daten gesichert und ausgewiesen ist;

- die Weiterverwendung der Daten für Forschung und Qualitätssicherung geregelt ist.

Die Empfehlungen sind rechtlich nicht verbindlich, jedoch durch eine sorgfältige Vernehmlassung und eine hohe Akzeptanz bei wichtigen Akteuren im Gesundheitswesen breit abgestützt. Die Empfehlungen werden von den herausgebenden Organisationen FMH, ANQ, $\mathrm{H}+$, SAMW und unimedsuisse periodisch evaluiert und bei Bedarf angepasst.

\section{Empfehlungen gesundheitsbezogene Register}

Die Empfehlungen zum Aufbau und Betrieb von gesundheitsbezogenen Registern sind als Flipbook und als PDF über www.saqm.ch $\rightarrow$ Publikationen $\rightarrow$ Empfehlungen 
Register oder unter folgendem Link abrufbar: http:// www.fmh.ch/saqm/publikationen/empfehlungenregister.html

Nachfolgend ist die Checkliste zur Überprüfung der gesundheitsbezogenen Register abgebildet.
Bei Fragen und weiteren Informationen stehen Ihnen die Herausgeberorganisationen FMH, ANQ, H+, SAMW und unimedsuisse gerne zur Verfügung (ddq[at]fmh. ch; 03135911 11).

\section{Checkliste zur überprüfung}

\section{Vorabklärungen bei der Planung eines Registers}

1.1 Der Bedarf/Nutzen des Registers ist für alle Beteiligten, auch für die Patientinnen und Patienten, beschrieben.

1.2 Die rechtlichen Rahmenbedingungen sind abgeklärt.

1.3 Die Einbettung des Registers ist abgeklärt.

1.4 Der Kontext sowie allfällige Interessenbindungen sind transparent aufgezeigt.

1.5 Der Aufbau und die längerfristige Finanzierung sind gesichert, ein Finanzierungskonzept liegt vor.

\section{Konzeption des Registers}

2.1 Zielsetzung und Aufgaben sind klar definiert sowie die damit verfolgten Fragestellungen formuliert.

2.2 Die Registerorganisation ist in einem Konzept/Reglement klar dargestellt.

\section{Kompetenzen zur Registerführung}

3.1 Die Kompetenzen der Registerführenden stimmen mit der Zielsetzung überein.

3.2 Die inhaltlich fachliche Kompetenz ist gesichert (wissenschaftliche Methodenkompetenz, klinische Kompetenz zum entsprechenden Fachgebiet).

3.3 Die technische Kompetenz ist vorhanden (Registeraufbau, Prozess, Logistik, Qualität und Sicherheit der Datenbank).

\section{Datenschutz und Datenhoheit}

4.1 Es ist ein Datenreglement vorhanden, das folgende Aspekte regelt:

a) Persönlichkeitsschutz: Beschreibung der Prozesse der Anonymisierung bzw. Verschlüsselung der Daten, Patienteninformation und -einwilligung sowie Einsichtsrecht, Umgang mit Widerruf und Datenaufbewahrung.

b) Datenzugang/Datenhoheit/Einsicht- und Zugriffsrechte/Weiterverwendung der Daten durch Dritte. 


\section{Datenerhebung und Datenverwendung}

5.1 Die zu erhebenden Variablen sind klar definiert und auf die Zielsetzung abgestimmt.

5.2 Die technischen Strukturen sind adäquat vorhanden und entwickelbar.

5.3 Der Anschluss an administrative/amtliche Daten bzw. die Möglichkeit der Integration in die Klinikinformationssysteme (Interoperabilität) sind gewährleistet.

5.4 Es liegt ein Datenflussdiagramm vor, welches die Datenerhebung, Datenübermittlung und Datenverarbeitung nachvollziehbar beschreibt.

5.5 Es liegt ein Auswertungskonzept vor, welches die Datenanalyse und die Darstellung der Ergebnisse präzise beschreibt.

5.6 Es liegt ein Publikationskonzept vor, welches Anforderungen, Inhalte und Form der Publikation sowie die Zielgruppen präzise beschreibt.

5.7 Die Weiterverwendung der Daten für Forschung wird unterstützt.

\section{Qualitätssicherung}

6.1 Es liegt ein Validierungskonzept inkl. periodischer Verfahren zur überprüfung vor, welches die Sicherstellung der Datenqualität gewährleistet.

6.2 Die Zielsetzungen und Aufgaben des Registers werden periodisch betreffend ihrer Zweckmässigkeit evaluiert.

\section{Zweckänderung und Auflösung}

7.1 Der Prozess bei einer Zweckänderung ist geregelt.

7.2 Der Prozess bei einer Registerauflösung ist geregelt. 